\title{
The role of laparoscopic dissection of the uterine artery in the surgical treatment of fibroid-related menorrhagia: a pilot study
}

Published online: 15 May 2004

(C) Springer-Verlag Berlin / Heidelberg 2004

\begin{abstract}
This pilot study assessed the outcome, tissue trauma, clinical improvement and reduction in size of fibroids following laparoscopic dissection of the uterine artery (LDUA) in fibroid-related menorrhagia. Fiftythree patients with complete records were included in the prospective clinical study. Before LDUA, and 3 and 6 months following the procedure, ultrasonography or MRI was done to measure the size of the uterus and dominant fibroid. Blood samples for hemoglobin and assay of marker inflammatory response and tissue trauma were taken preoperatively on the 1st and 3rd postoperative days. All patients underwent successful LDUA using ultrasonically activated shears without intra-operative complications. Fifty (96.2\%) laparoscopically treated patients with fibroids who subsequently experienced improvement in menorrhagia and anemia are described. The LDUA procedure can be completed within 30-40 min with only minimal blood loss and a short hospital stay if performed by experienced surgeons. Our study results show that single laparoscopic dissection of the uterine artery performed with ultrasonic technique is associated with an insignificant stress response. The average reductions in the uterine volume and dominant fibroid volume were 36.6 and $57.9 \%$ at 6 months after surgery, respectively. Four women conceived within 1 year, and their pregnancies were without complications during gestation.
\end{abstract}

\footnotetext{
Z. Holub $(\bullet) \cdot$ L. Kliment · J. Lukac · S. Urbanek

Department of Obstetrics and Gynecology,

Endoscopic Training Center,

Baby Friendly Hospital,

Vancurova Street 1548, 27258 Kladno, Czech Republic

e-mail: holubz@seznam.cz

Tel.: +42-312-606417

Fax: +42-312-606417

\section{A. Jabor}

Department of Biochemistry and Statistical Analysis,

Baby Friendly Hospital,

Kladno, Czech Republic
}

Keywords Uterine fibroids · Menorrhagia $\cdot$ Laparoscopic dissection of uterine artery

\section{Introduction}

Menorrhagia, defined as menstrual blood loss of $80 \mathrm{ml}$ or more in a cycle, is a common problem among women of reproductive age [1]. If uterine fibroids are causing menorrhagia with anemia, then medical or surgical treatment is appropriate. Surgery for menorrhagia is generally utilized when medical therapy fails, it is not tolerated by the woman, or because of patient or physician choice. Uterine fibroids can be treated by myomectomy, myolysis, uterine artery embolization (UAE) or hysterectomy [2]. Laparoscopic bipolar coagulation and dissection uterine vessels were recently considered the treatments choice [3, 4]. A systematic search of the biomedical database Medline using the terms "uterine artery coagulation," "ligation" or "occlusion and menorrhagia" only identified case reports or non-randomized studies [3, 5, 6, 7]. The aim of this pilot study was to assess prospectively the effectiveness and safety of laparoscopic dissection of the uterine artery (LDUA) in the management of menorrhagia in women with uterine fibroids. The study is part of the prospective Czech multicenter study FSAT (Fibroid Surgery Alternatives Treatment).

\section{Materials and methods}

The approval of the local research ethics committee was obtained, and patients gave informed consent. The prospective pilot study was carried out at the Department of Gynecology and Obstetrics and Endoscopic Training Center at the Baby Friendly Hospital in Kladno, Czech Republic. During the period from January 2001 to June 2003, 98 consecutive selected women with symptomatic fibroids confirmed by ultrasound and/or MRI were treated with laparoscopic dissection of the uterine artery. The patients with submucosal fibroids were excluded. Fifty-five women presented with menorrhagia, which was considered the dominant symptom and the main inclusion criterion. Uterine bleeding was assessed from the menstrual flow diary recorded by the patients. Menorrhagia was 


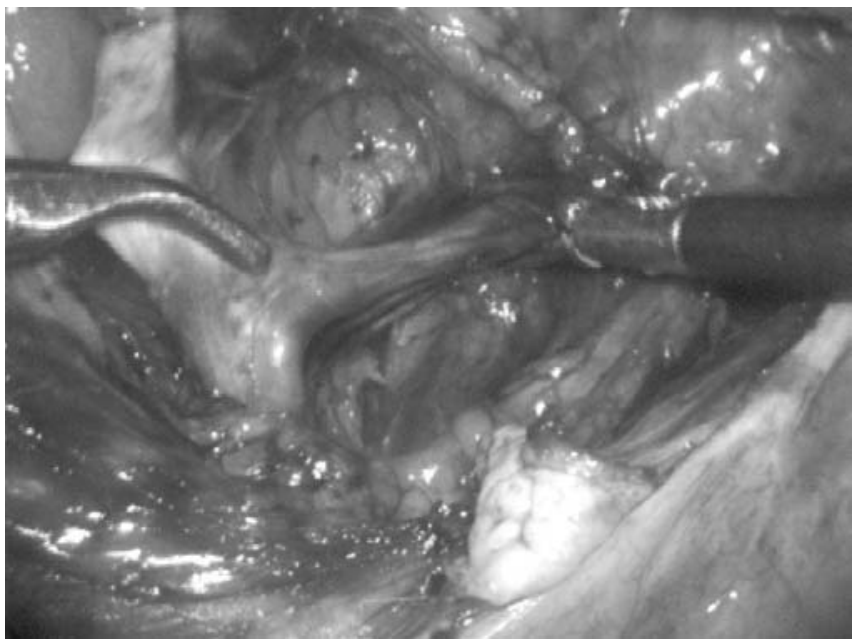

Fig. 1 Skeletonizing the left uterine artery before dissection. The grasper coming from top right lifts the uterine artery from the ureter

diagnosed if it had been present for 3 months or more in the previous 6 months or required seven saturated pads or more per day. In the absence of hematologic disorders, a hemoglobin level below $120 \mathrm{~g} / \mathrm{dl}$ was classified as anemia in 39 patients $(73.5 \%)$. Fifty-three patients with complete records were included in the study. Before LDUA and 3 and 6 months following the procedure, ultrasonography or MRI was carried out to measure the size of the uterus and dominant fibroid. The volume of the size of the uterus and dominant fibroid was calculated measuring the maximal anterior-posterior, transverse and sagittal diameters and multiplying the product of these by 0.52 before and after uterine artery dissections [8].

All patients had normal preoperative FSH, LH, 17 $\beta$-estradiol, C reactive protein (CRP), cortisol, creatin kinase (CK) levels and white blood cell (WBC) counts without a significant concomitant disease. All women required either hysterectomy or myomectomy and wished to keep their uterus. Fourteen women wished to achieve pregnancy. Duration of the surgical procedure was calculated from the skin incision to the last skin suture. The intra-operative blood loss was measured as blood volume accumulated in the aspirator during the operation. The postoperative blood loss was approximated from the postoperative fluid collection using Redon's catheter. Prophylaxis consisted of a single dose of ampicillin $(2.0 \mathrm{~g})$ and anticoagulant therapy with low molecular-weight heparin.

Blood samples for hemoglobin and assay of markers inflammatory response and tissue trauma (CRP, cortisol, CK, WBC) were taken preoperatively on the 1 st and 3 rd postoperative days. The hemoglobin was assessed 3 months postoperatively as well. Patients were interviewed 3 and 6 months after surgery regarding changes in bleeding and other fibroid bulk-related symptoms on a scale described by Yen et al. [9].

Operative procedure

Laparoscopy was performed in the lithotomy position using videomonitoring equipment. The telescope was inserted subumbilically and one 11-mm port was made suprapubically and medially. Two 5-mm ports were placed in each of the lower quadrants at the lateral edge of the rectus abdominis muscle. Lateral peritoneum dissection using ultrasonically activated shears (LCS-K5, Ultracission, Ethicon EndoSurgery, Johnson\&Johnson, Ltd., Cincinnati) was started. The line of dissection was between the round ligament anteriorly, infundibulopelvic ligament medially and pelvic sidewall laterally. The peritoneum was opened and the paravesical and obturator space developed by blunt dissection. Subsequently, distancing uterine vessels from the ureter with a safe ultrasonic coagulation of the uterine artery and cutting just medial to their origin from the hypogastric artery were performed (Fig. 1). In selected cases of subserosal fibroids, myomectomy using LCS or the harmonic scalpel followed.

\section{Statistical analysis}

Statistical analysis was performed using the Kruskal-Wallis test one-way analysis of variance (ANOVA). Data are presented as the median, standard deviation (SD) and range or as the number or percentage. Statistical significance was defined as $P<0.05$.

\section{Results}

The characteristics of the women are shown in Table 1 . Laparoscopic lateral dissection of the uterine artery was performed successfully in all cases. All patients underwent a successful operative procedure and completed follow-up at 3 and 6 months. At the time of writing, four women $(28.5 \%)$ had successfully conceived and two of them had delivered at term. Of the two term pregnancies, one was delivered vaginally, and the other was delivered via cesarean section

\section{Surgical results}

We elected to desiccate the anastomosis zone of the uterine arteries and ovarian arteries with ultrasonic shears in the first patient only. Subserosal myomectomy and uterine artery dissection were performed successfully in 21 women $(39.6 \%)$. The main indication for surgery was menorrhagia, but most patients $(57 \%)$ had more than one indication. Values related to the number of fibroids, number and size of enucleated fibroids and largest fibroid diameter are shown in Table 1. The size of the dominant fibroid ranged from 3 to $8 \mathrm{~cm}$. The mean of the dominant fibroid was $5.7 \mathrm{~cm}$. The size of the removed fibroids ranged from 1 to $6 \mathrm{~cm}$ (mean $3.9 \mathrm{~cm}$ ).

Suspicious extra-uterine endometriosis implantations in the uterosacral ligament, fibroid and cul-de-sac were found in $7.5 \%(n=4)$ of all patients. The time of surgery ranged from 15-50 min (mean $40 \mathrm{~min}$ ) in cases of LDUA. If the LDUA was combined with myomectomy, the mean time of surgery was 77.9 min (range, 30-120 min). Mean blood loss was less than $25 \mathrm{ml}$. The women were given one to two doses of intramuscular metamidazolum ni-

Table 1 Characteristics of women and fibroids

\begin{tabular}{lcc}
\hline Mean age (years) (range) & 36.4 & $24-50$ (range) \\
Mean weight (kg) (range) & 64.0 & $51-98$ (range) \\
Previous pelvic surgery- $n$ & 7 & $13.2 \%$ \\
No. of fibroids in each women & 48 & $90.5 \%$ \\
$\quad 1-3$ & 5 & $9.5 \%$ \\
$>3$ & 31 & $58.5 \%$ \\
Size of fibroids (largest diameter, cm) & 22 & $41.5 \%$ \\
$<5.9$ & 21 & $39.6 \%$ \\
$>6.0$ & 5.7 & $3-8$ \\
No. of enucleated fibroids & \\
Size of enucleated fibroids (mean, range, & \\
$\mathrm{cm}$ ) & &
\end{tabular}


Table 2 LUAD results, clinical improvement

\begin{tabular}{lrll}
\hline Length of follow-up (months) & Significantly improved & Slightly improved & Unchanged \\
\hline Menorrhagia $(n=53)$ & 49 & 2 & 2 \\
3 months & 50 & 1 & 2 \\
6 months & 23 & 3 & 5 \\
Pelvic pain or other symptoms $(n=31)$ & 2 & 3 \\
3 months & 26 & & \\
6 months & & & \\
\hline
\end{tabular}

Table 3 Results of hemoglobin and tissue markers

\begin{tabular}{lcccll}
\hline & Preoperative & 1 POD & 3 POD & 3 months & $P$ value \\
\hline Hemoglobin & $120.0 \pm 12$ & $1,116.7 \pm 10.1$ & $115.3 \pm 12.8$ & $139 \pm 8.2$ & $<0.01$ \\
CRP $(\mathrm{mg} / \mathrm{l})$ & $5.0 \pm 1.9$ & $10.0 \pm 26.4$ & $9.0 \pm 32.2$ & - & $<0.05$ \\
CK $(\mu \mathrm{kat} / \mathrm{l})$ & $1.1 \pm 0.3$ & $2.7 \pm 0.4$ & $1.9 \pm 0.7$ & - & $\mathrm{NS}$ \\
Cortisol $(\mathrm{nmol} / \mathrm{l})$ & $515.5 \pm 104.0$ & $483.0 \pm 78.5$ & $529.0 \pm 97.0$ & - & $\mathrm{NS}$ \\
WBC $\left(10^{9} / \mathrm{l}\right)$ & $6.4 \pm 1.4$ & $8.4 \pm 1.7$ & $7.3 \pm 2.1$ & - & $<0.01$ \\
\hline
\end{tabular}

tricum (1.0 g) daily to relieve pain after surgery. One major postoperative complication occurred: in one case, port site intra-abdominal bleeding was observed $6 \mathrm{~h}$ after surgery. The port site bleeding was successfully treated by relaparoscopy, Foley catheter and transfusion. Minor postoperative complications were as follows: one postoperative wound infection and two cases of fever over 3 days. The patients were discharged the 2nd day after surgery, and the mean hospital stay was 2.5 days. They returned to work 2 weeks later. Twenty-one women experienced lower abdominal pain postoperatively, which usually developed 5-7 days after surgery.

\section{Clinical improvement}

The results of the clinical improvement are shown in Table 2. A significant improvement rate of menorrhagia was $92.4 \%(49 / 53)$ and $96.2 \%(50 / 53)$ at 3 and 6 months, respectively. In one woman with slight improvement of menorrhagia, the preoperative blood level of hemoglobin was increased significantly from $123 \mathrm{~g} / \mathrm{l}$ before LUAD to $133 \mathrm{~g} / \mathrm{l}$ after 3 months. At times, the pelvic pain or bulk-related symptom improvement rates were 83.8 and $90.3 \%$. In two women with endometriosis, the recurrence of the pelvic pain was found 4 months after surgery.

Hemoglobin and tissue marker results

The preoperative blood hemoglobin level significantly increased from a mean of $120 \mathrm{~g} / \mathrm{dl}$ before LDUA to a mean of $139 \mathrm{~g} / \mathrm{dl}$ after 3 months $(P<0.01)$, (Table 3$)$. The insignificant decline in the hemoglobin level was $0.47 \mathrm{~g} / \mathrm{dl}$ on the $3 \mathrm{rd}$ postoperative day. Preoperative levels of studied markers were low in all cases, and the circulating concentrations of CRP and white blood cells increased significantly after surgery $(P<0.05$ and $P<0.01$, respectively). When the values for LDUA alone and LDUA combined with myomectomy were analyzed separately, a significant increase of CRP and WBC levels $(P<0.01)$ was found only for the combined surgical pro- cedures. The plasma concentrations of cortisol and creatine kinase increased insignificantly during the first 3 postoperative days. All patients had normal preoperative FSH, LH and $17 \beta$-estradiol levels. Only one perimenopausal woman with coagulated anastomotic sites of uterine and ovarian vessels reported irregular bleeding and hot flushes after surgery. The level of FSH was 46 IU $\mathrm{ml}$, LH $14 \mathrm{IU} \mathrm{ml}$ and $17 \beta$-estradiol $30 \mathrm{pg} \mathrm{ml}$. Within 1 month, the vasomotor symptoms decreased after estrogen therapy. Three months later, the visit showed that the patient's symptoms were completely resolved, and the FSH was $34 \mathrm{IU} \mathrm{ml}$ with a normal estrogen level.

\section{Sonographic follow-up}

The maximum diameter of the fibroids ranged from 2 to $8 \mathrm{~cm}$. Twenty-one women (39.6\%) had fibroids with a diameter over $6 \mathrm{~cm}$. Forty-three (81\%) women had multiple fibroids. Pretreatment pelvic ultrasound in 53 women showed uterine enlargement, with average dimensions of $11.5 \mathrm{~cm} \times 7.1 \times 7.6 \mathrm{~cm}$, with a calculated volume of $322.68 \mathrm{~cm}^{3}$ (range $93.60-598.48 \mathrm{~cm}^{3}$ ). The average reductions in uterine volume were 24.1 and $36.6 \%$ at 3 and 6 months, respectively. The average size of the dominant was $5.6 \times 5.4 \times 5.2 \mathrm{~cm}$, with a calculated volume of $81.7 \mathrm{~cm}^{3}$ (range $27.0-240.24 \mathrm{~cm}^{3}$ ). The average reductions in the volume of the dominant fibroids were 28.8 and $57.9 \%$ using the prolate ellipse equation. In 21 women with LDUA and myomectomy, the removed subserosal fibroids were not dominant fibroids.

\section{Discussion}

Uterine fibroids and adenomyosis are the most common benign pathologies of the female pelvis and are responsible for symptoms such as menorrhagia, dysmenorrhea, pelvic pain and urinary frequency. The choice of surgical alternative treatments for symptomatic fibroids is dependent on their location within the uterus [10]. Submucosal fibroids may be effectively treated hysteroscopically, 
whereas subserosal and intramural fibroids will require an abdominal, laparoscopic or vaginal approach. Myomectomy is the surgical option of choice for women who wish to retain their uterus. Hysterectomy is one of the few surgical procedures that has a $100 \%$ primary success rate for the presenting problem of menorrhagia [1]. Recently, uterine artery embolization (UAE) has proved to be highly effective in controlling fibroid-related bleeding and initiating tumor degeneration [11]. The technique of UAE resulted in fibroid shrinkage by 39 to $70 \%$ and a clinical symptoms response rate of 85 to $90 \%$, but it is limited by radiation exposure, ovarian failure, postembolization syndrome and potentially fatal complications after treatment [12]. As an alternative method of obstructing the uterine blood flow, uterine artery ligation should also be considered. Liu et al. [3] and Holub et al. [4] reported the effective management of myoma with laparoscopic bilateral uterine artery coagulation and dissection, respectively. Nevertheless, the exact mechanism of uterine artery embolization and ligation involved in reducing fibroid size has not been identified clearly. The results study of Park et al. [13] suggested that laparoscopic uterine artery ligation results mainly in physiological cell death, or apoptosis, whereas the corresponding result is cell necrosis for UAE.

The initial complaint of menorrhagia is a subjective one and comes from the women's perceived menstrual loss. There is a marked variation in a woman's sensitivity to this degree of loss, with many women either over- or under-reporting their menstrual loss [1]. In a study in women with iron-deficiency anemia because of menorrhagia, only $15 \%$ described their periods as heavy [14]. At the other end of the spectrum, it has been reported that 26-38\% of women complaining of heavy periods had objective menstrual losses of less than $80 \mathrm{ml}$ per cycle [15]. In our study, we found that $26.5 \%$ of women complaining of menorrhagia had normal blood hemoglobin levels before surgery. On the other hand, we observed that the postoperative hemoglobin level increased in one woman, which was not satisfied with uterine artery dissection treatment of menorrhagia. Symptom improvement was evaluated by patients' subjective reporting, so that a placebo effect could be present in some patients.

Our previous case report experience was applied to 53 cases of uterine artery dissection in women with menorrhagia and uterine fibroids [4]. All patients underwent successful LDUA with low rates of postoperative complications. One woman underwent relaparoscopy for postoperative port site intra-abdominal bleeding. Two women developed fever in the 1st week after surgery and were treated with antibiotics. Fifty $(96.2 \%)$ women with menorrhagia reported a satisfactory decrease of bleeding at 6 months after uterine artery dissection. All 36 patients with anemia had blood hemoglobin levels over $120 \mathrm{~g} / \mathrm{dl}$ after 3 months. The postoperative clinical improvement rate for pelvic pain and other bulk-related symptoms was 83.8 and $90.3 \%$ at 3 and 6 months, respectively. The technique of LDUA resulted in fibroid shrinkage. Signif- icant increases of CRP and WBC were found only for LDUA combined with myomectomy.

Our study results show that single laparoscopic uterine artery dissection performed ultrasonically activated shears is associated with insignificant stress response. The ultrasonic operative technique provides both hemostasis and cutting with decreased charring of tissue and less thermal damage surrounding the myometrial structures, allowing better healing because there is less need to evacuate smoke from the abdomen cavity [16, 17].

One of the most important issues for laparoscopic uterine artery occlusion or UAE involves adequacy of approach for women who wish to achieve pregnancy. There are few studies about this in the literature [7, 11, $18,19]$. Chen et al. [7] reported that the pregnancy and term pregnancy rates in sexually active women without contraception were 41.6 and $5.6 \%$, respectively. The relatively high rate of $41.2 \%$ for early miscarriages was observed. Pregnancy following UAE has been reported. The largest number of pregnancies from one center was reported by Ravina et al. [11]. They noted 12 pregnancies in women aged 22 to 41 years. Of these, five resulted in a miscarriage and three in pre-term deliveries. This high rate of miscarriage is a cause for concern. Although it has not been reported, the decrease in uterine blood flow after UAE can also lead to intrauterine growth restriction [4, 11]. However, the effect of uterine artery embolization in pregnancy is unknown because, in theory, UAE might directly affect endometrial and myometrial perfusion [7].

In the present study, 14 women planned to achieve pregnancy in 1 to 3 years. Four women conceived within 1 year, and their pregnancies were without complications during gestation at the time of writing. According to the term of delivery, the infants' birth weights were normal. In contrast to the operative procedure reported by Liu et al. [3], LDUA does not influence uterine blood supply from the ovarian artery. The issue of pregnancy outcome after uterine artery occlusion in UAE, laparoscopic coagulation and dissection of the uterine artery need to be studied prospectively in large randomized study.

\section{Conclusion}

Fifty $(96.2 \%)$ of 53 laparoscopically treated patients with fibroids who subsequently experienced improvement in menorrhagia and anemia with low rates of complications are described. The lateral dissection of the uterine artery using the ultrasonic operative technique is a feasible surgical procedure. The LDUA procedure can be completed within 30-40 min with only minimal blood loss and a short hospital stay if performed by experienced surgeons. We consider the treatment using laparoscopic dissection of the uterine artery to be a promising surgical procedure for women with fibroid-related menorrhagia. We still need to assess the effect on subsequent pregnancy, the long-term results of this new surgical technique and possible side effects. 


\section{References}

1. Abbot JA, Garry R (2002) The surgical management of menorrhagia. Hum Reprod Update 8:68-78

2. Lefebre G, Vilos G, Allaire C, et al (2003) SOGC clinical practice guidelines. The management of uterine leiomyomas. J Obstet Gynecol Can 128:396-405

3. Liu WM, Ng HT, Wu YC, et al (2001) Laparoscopic bipolar coagulation of uterine vessels: a new method for treating symptomatic fibroids. Fertil Steril 75:417-422

4. Holub Z, Lukáč J, Kliment L, Urbánek Š (2003) Short-term results from laparoscopic dissection of uterine vessels in women with fibroids. Eur J Obstet Gynecol Reprod Biol 110:94-98

5. Mitchell GG, Mellor S, Burslem RW, et al (1977) Pregnancy following uterine artery ligation. Br J Obstet Gynecol 84:551554

6. Lichtinger M, Hallson L, Calvo P, Adeboyejo G (2002) Laparoscopic uterine artery occlusion for symptomatic leiomyomas. J Am Assoc Gynecol Laparosc 9:191-198

7. Chen YJ, Wang PH, Yuan CHCH, et al (2003) Pregnancy following treatment of symptomatic myomas with laparoscopic bipolar coagulation of uterine vessels. Hum Reprod 18:10771081

8. Goldstein SR, Horii SC, Snyder JR, et al (1998) Estimation of nongravid uterine volume based on nomogram of gravid uterine volume: its value in gynecological abnormalities. Obstet Gynecol 72:86-90

9. Yen YK, Liu WM, Yuan CH, et al (2001) Laparoscopic bipolar coagulation of uterine vessels to treat symptomatic fibroids with elevated CA 125. J Am Assoc Gynecol Laparosc 8:241246

10. Falcone T, Bedaaiwy MA (2002) Minimally invasive management of uterine fibroids. Curr Opin Obstet Gynecol 14:401407

11. Ravina JH, Vigneron NC, Le Dref O, et al (2000) Pregnancy after embolization of uterine myoma: report of 12 cases. Fertil Steril 73:1241-1243

12. Payne JF, Haney AF (2003) Serious complications of uterine artery embolization for conservative treatment of fibroids. Fertil Steril 79:128-131

13. Park KH, Kim JY, Shin JS, et al (2003) Treatment outcomes of uterine artery embolization and laparoscopic uterine artery for uterine myoma. Yonsei Med J 44:694-702

14. McKenna DM, Dockeray CJ, McCann (1989) Iron deficiency anaemia in pre-menopausal women. Ir Med J 82:69-70

15. Fraser IS, McCarron G, Markham R (1984) A preliminary study of factors influencing perception of menstrual blood loss volume. Am J Obstet Gynecol 149:788-793

16. Stringer NH (1994) Laparoscopic myomectomy with harmonic scalpel: a review of 25 cases. J Gynecol Surg 10:241-244

17. Koh C, Janik G (2003) Laparoscopic myomectomy: the current status. Curr Opin Obstet Gynecol 15:295-301

18. Chen YJ, Wang PH, Yuan CC, et al (2002) Successful pregnancy in a woman with symptomatic fibroids who underwent laparoscopic bipolar coagulation of uterine vessels. Fertil Steril $77: 838-840$

19. Chen YJ, Wang PH, Yuan CC, Wu YC, Liu WM (2002) Early pregnancy uninterrupted by laparoscopic bipolar coagulation of uterine vessels. J Am Assoc Gynecol Laparosc 9:79-83 